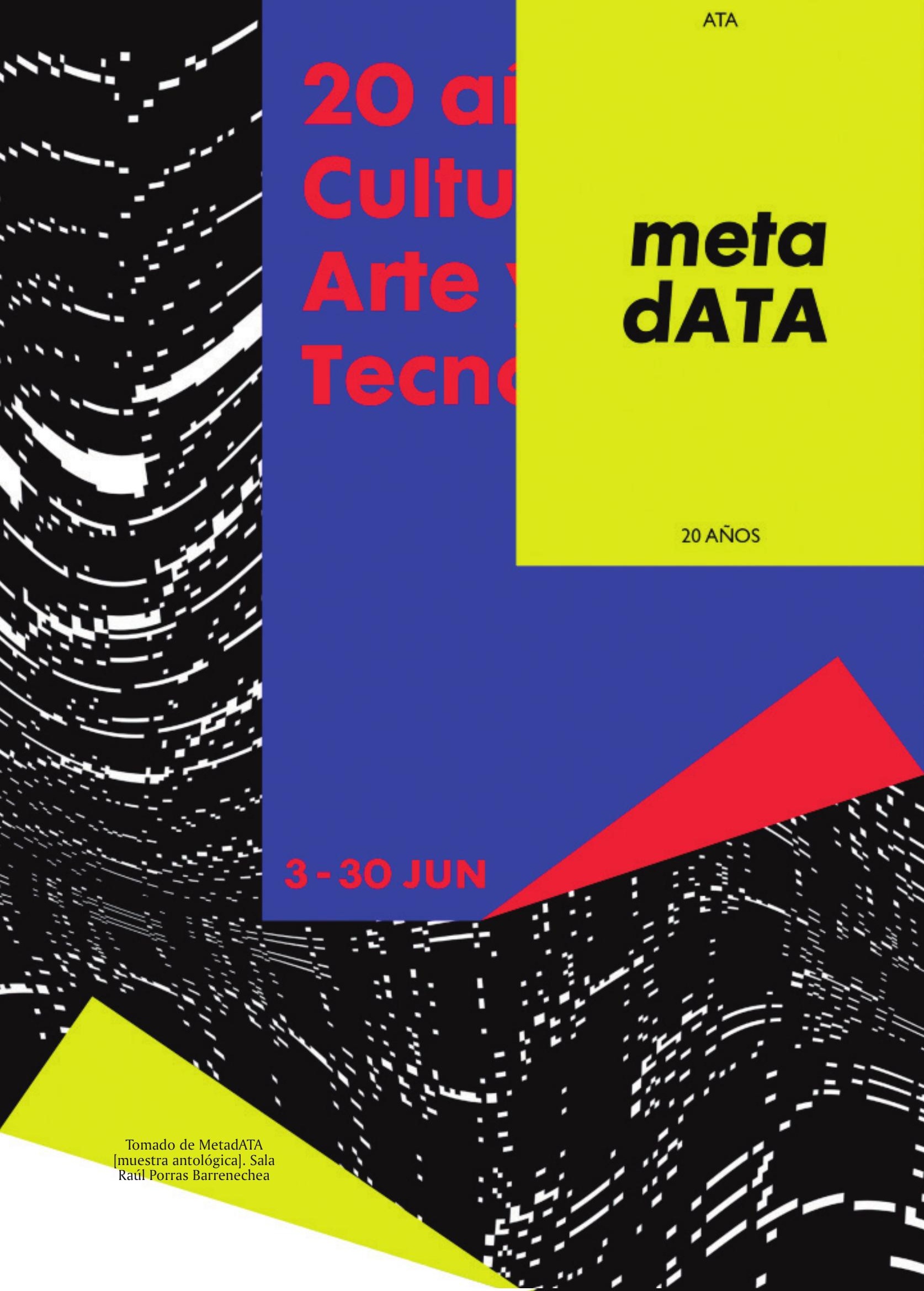




\title{
Recreando artefactos digitales: del archivo de ATA a la curaduría de metadATA
}

\author{
José-Carlos Mariátegui / Nataly Montes Becerra \\ Alta Tecnología Andina \\ jcm@ata.org.pe / montesb.nataly@gmail.com
}

\begin{abstract}
Resumen
Este artículo intenta describir críticamente la exhibición metadATA: 20 años de cultura, arte y tecnología, muestra antológica de la organización Alta Tecnología Andina (ATA), presentada del 3 al 30 de junio del 2016 en el Centro Cultural Ricardo Palma de la Municipalidad de Miraflores. Este proyecto buscaba decantar, a partir de seis hitos temáticos en su historia desde su fundación, en los años noventa, una parte importante de la historia del arte y la tecnología en el Perú. En primer lugar, presentamos una breve historia del contexto histórico y la necesidad de producir esta muestra. Luego analizamos el concepto curatorial, así como las seis cápsulas de información propuestas. Posteriormente, incidimos sobre la museografía y el diseño de la exhibición, y los dispositivos tecnológicos empleados. Concluimos con un análisis sobre la complejidad conceptual en relación con la práctica curatorial en archivos y su nivel de objetividad en el presente.
\end{abstract}

Palabras clave: curaduría, archivos, museografía, Perú, exhibición, arte, tecnología

\begin{abstract}
This article tries to critically review the exhibition "metadATA: 20 years of culture, art and technology", an anthological exhibition of the work done by Alta Tecnología Andina (ATA). It was showed from the 3rd to 30th of June 2016 at the Centro Cultural Ricardo Palma of the Miraflores City Council. This exhibition sought to showcase, from six thematic milestones, a relevant part of the history of art and technology in Peru since the 1990s. First we give a brief history on the historical context and the need to present such exhibition. Then we analyze the curatorial concept as well as the organization of the six information capsules. Subsequently, we focus on the museography and the exhibition design as well as the importance of the technological devices. We conclude with an analysis the philosophical complexity of the producing curatorial work of previously curated work and the relevance in the present.
\end{abstract}

Keywords: Curating, archives, museography, Peru, exhibition, art, technology

\section{Contexto histórico y urgencia}

A partir de la década de 1970, aparecieron de manera esporádica obras y exhibiciones de video y nuevos medios en el escenario de las artes visuales peruanas. Estos trabajos continuaron en los años ochenta, aunque quedan pocos registros de dicha etapa. En cuanto a los artistas, podemos mencionar entre los principales exponentes a Francesco Mariotti, 
Rafael Hastings, Teresa Burga, Esther Vainstein, Mario Acha, Mario Pozzi Escot o Juan Javier Salazar. Sin embargo, no fue hasta mediados de los años noventa que pudo hablarse de una actividad continua y sostenida en la videocreación en el Perú, a la que se sumó el desarrollo del arte electrónico de forma creciente y significativa a finales de dicha década ${ }^{1}$.

Justamente es en 1995 cuando Alta Tecnología Andina (ATA) inicia su apoyo a la producción de proyectos de video y el arte electrónico en nuestro medio; a partir de 1998, el reinicio del Festival Internacional de Video/Arte/Electrónica, en Lima, permitió el establecimiento de un nuevo y fructífero campo dentro de las artes visuales peruanas que catalizó nuevos modelos de trabajo basados en la colaboración y el intercambio entre organizaciones y personas tanto a nivel local como internacional. Estas interacciones venían de la mano con una serie de actividades que generaron contactos internacionales e invitaciones para realizar curadurías de carácter itinerante a nivel internacional. A ello siguió, desde 2005, la investigación y descubrimiento de artistas peruanos que trabajaban en el extranjero sin contacto con el país, pero relevantes en el contexto de las artes visuales globales y luego con proyectos vinculados a las relaciones entre cultura, tecnología y sociedad. Gracias a ATA, como plataforma, se han podido crear nuevos vínculos con escenarios globales, que hoy resultan significativos para la historia de las artes visuales en el Perú.

Sin embargo, la escasa información disponible sobre el desarrollo de los nuevos medios en la creación artística en el Perú y el desconocimiento de los agentes y autores significativos en este panorama no permiten establecer un panorama histórico coherente.

Por ello, conmemorando los veinte años de ATA, realizamos un trabajo de investigación en sus archivos, que permitió recopilar, analizar y catalogar importantes materiales que resumen dos décadas de iniciativas y proyectos sobre el video y las artes electrónicas en el Perú, organizadas por ATA y gestadas de la mano de un grupo humano integrado por investigadores, artistas, tecnólogos, comunicadores e historiadores, que son un referente en el desarrollo de la cultura, el arte y la tecnología de las dos últimas décadas en la forma de festivales, exhibiciones, producciones, debates y colaboraciones en el Perú, América Latina y el mundo.

\section{Protoprocesos: investigando el archivo, conceptualizando la exhibición}

La primera etapa del proceso consistió en recopilar, analizar, catalogar y organizar, a partir de la revisión exhaustiva, el conjunto de materiales correspondiente a los proyectos en los que se había involucrado ATA desde diversos roles (como organizador, colaborador, productor y asesor) y de la mano con diferentes actores y organizaciones, tanto locales como extranjeras. Se definieron seis temas clave que incluiría la muestra y que fueron denominados cápsulas de información: historia de los inicios de ATA, compilación de las ediciones del Festival Internacional de Video/Arte/Electrónica, selección de proyectos con alcance internacional, selección de colaboraciones con organizaciones y personas, el proyecto Escuelab $\mathrm{y}$, finalmente, un repositorio dedicado a trabajos audiovisuales.

Después del diseño esquemático de información basado en la investigación del archivo, se recopilaron diversos tipos de insumos en la forma de documentos, material gráfico, libros, cintas de video y fotografías, que nos permitieron trabajar progresivamente en el análisis de las piezas, la catalogación y la organización de material. El material era diverso e incluía registros o filmaciones de limitada difusión o que, por primera vez, serían presentadas públicamente. Este trabajo de filtro y revisión, que tomaría cerca de cuatro meses, fue muy importante, ya que permitió organizar y catalogar el material, así como digitalizar y editar

1 Para mayor información sobre esta etapa, revisar el artículo Mariátegui, J.-C. (2008). Días de videoarte. Una intensa década de videoarte en el Perú. ILLAPA Mana Tukukuq. Revista del Instituto de Investigaciones Museológicas y Artísticas de la Universidad Ricardo Palma, 5, pp. 53-62. 


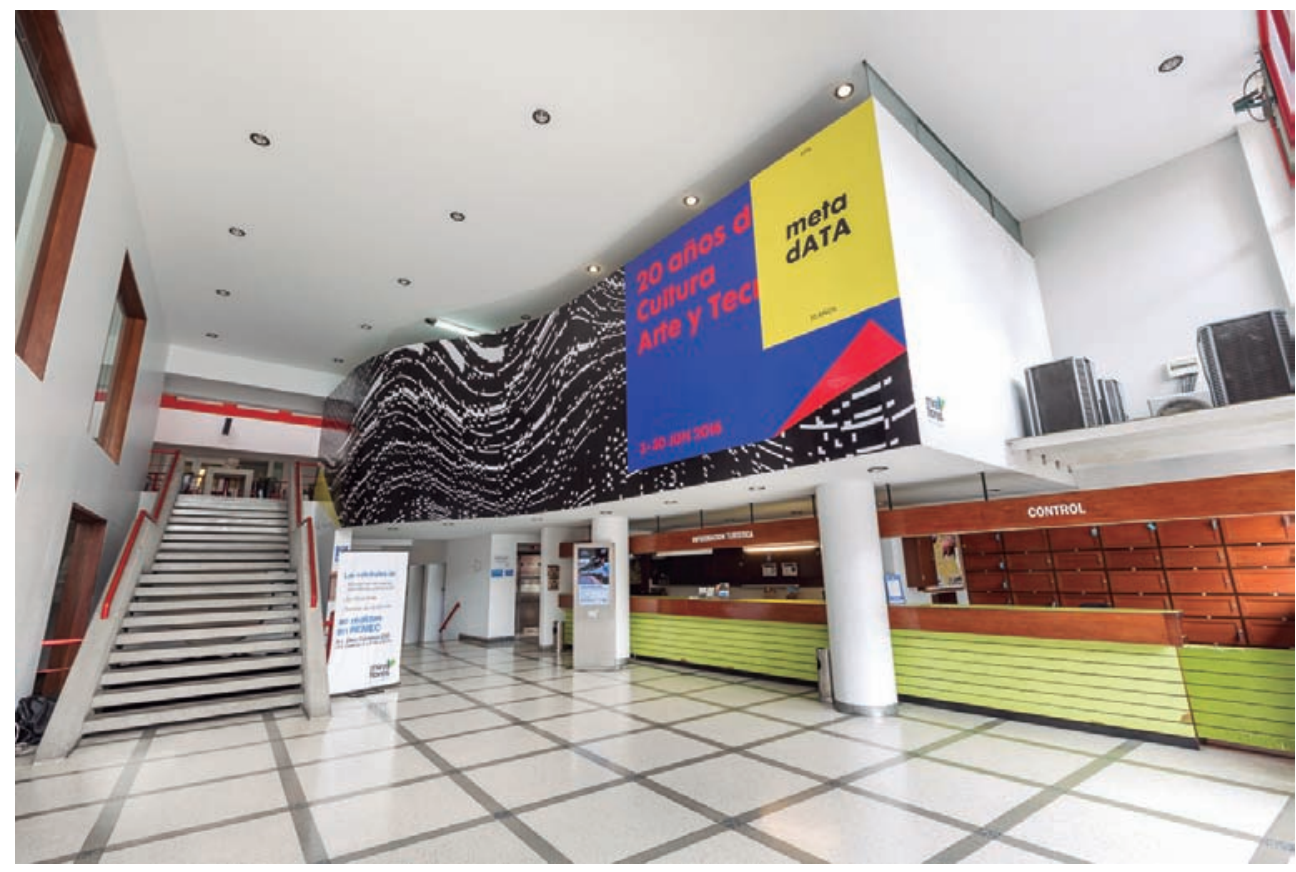

Fig. 1. MetadATA [muestra antológica] . Sala Raúl Porras Barrenechea. Visita junio del 2016. Foto: Wilber Dueñas.

videos que serían presentados o servirían como insumo para generar nuevos contenidos audiovisuales que, de manera didáctica, permitirían describir los proyectos seleccionados.

La muestra tuvo lugar en las dos galerías públicas, ubicadas en el Centro Cultural Ricardo Palma de la Municipalidad de Miraflores (Fig.1): la Sala 770 y Sala Raúl Porras Barrenechea, espacios dedicados a la exhibición y difusión de arte contemporáneo peruano y latinoamericano. En suma, ambos espacios, reciben entre 8000 a 10000 visitantes por mes y están abiertos al público todos los días del año.

Debido a la amplitud del público visitante, la construcción de la muestra siempre estuvo pensada en brindar muchas herramientas esquemáticas y narrativas (storytelling) para la comunicación de los diferentes estadios en la historia de ATA. Sabíamos que el tiempo que se tendría que dedicar en recorrer todo el espacio supondría un reto para los visitantes, por la gran cantidad de información disponible. Es así que cada una de las cápsulas que componían la exposición eran independientes una de la otra, lo que permitía vivir la muestra por secciones o aspectos particulares que más interesaran a los visitantes (Fig. 2).

El constructo conceptual se creó por un equipo de discusión conformado por Reina Jara, José-Carlos Mariátegui, Nataly Montes y Jorge Villacorta. Desde los inicios, se veía el proyecto

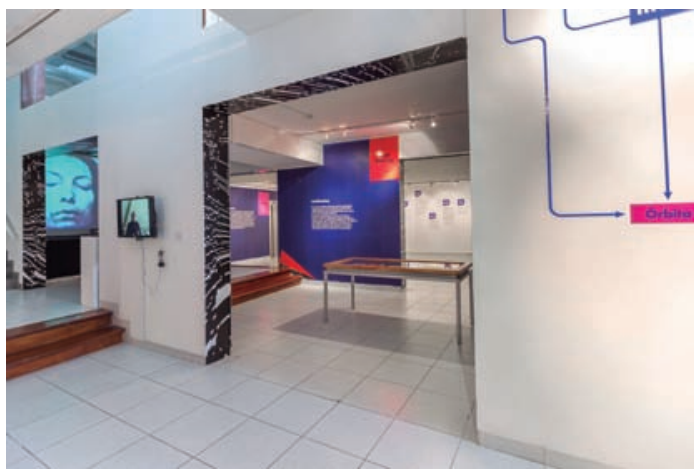

Fig. 2. MetadATA [muestra antológica] . Sala Raúl Porras Barrenechea. Visita junio del 2016. Foto: IS Creative Studio. 


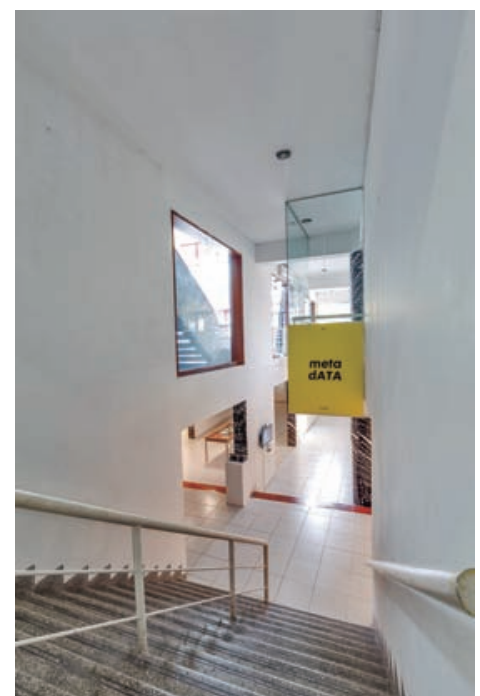

Fig. 3. MetadATA [muestra antológica]. Sala Raúl Porras Barrenechea. Visita junio del 2016. Foto: Wilber Dueñas. de exhibición como un gran repositorio de información estructurada o metadata. La metadata describe las características más importantes y distintivas de un recurso digital, compartiendo muchas similitudes con la catalogación realizada en bibliotecas; la producción de metadata permite acceder y buscar fácilmente a la información. Por ese motivo, la metadata, en particular para acceder al video, se ha vuelto un componente crucial en la producción de cualquier tipo de artefacto digital. Es así que surgió el juego de palabras metadATA (Fig.3), que eclipsó ambos aspectos que se necesitaban comunicar en la muestra, y se agregó un subtítulo explicativo. Finalmente, la muestra quedó titulada como metadATA, 20 años de Cultura, Arte y Tecnología.

\section{Cápsulas de información}

Como ya mencionamos, se definieron seis ejes temáticos que incluiría la muestra, que se denominaron cápsulas de información. A cada cápsula de información, se le asignó un título creado a partir de conceptos propios de los contenidos y temas que se iban incorporando. Es así como la historia de los inicios de ATA

se convirtió en ID-o-logo; el compilado de los festivales internacionales de videoarte, en Avanzada Digital; la selección de proyectos con alcance internacional, en Órbita; la selección de colaboraciones con organizaciones y personas, en Multitasking; el espacio dedicado a Escuelab, en LatAm; y el repositorio de proyectos audiovisuales, en VideodATA. A fin de vincular las cápsulas entre sí, formando un ecosistema de nodos interrelacionados, se armó un mapa conceptual que fue presentado visualmente como parte de la muestra (Fig. 4).

El primer eje, ID-o-logo, es un juego verbal entre el término ID de identificación y la palabra $\log o$, que se combinan en alusión verbal también a la definición ideológica que decantó en el manifiesto inicial de ATA (Fig. 5). Contiene documentos de los inicios de ATA, en particular la estrecha relación con el artista Gianni Toti, precursor internacional de la videopoesía y colaborador de la definición de ATA (1995), toda vez que el acto inaugural de la organización consistió de una retrospectiva de sus principales trabajos ${ }^{2}$, así como el evento por el centenario de M. C. Escher ${ }^{3}$, que derivó también en la creación del logo de ATA diseñado especialmente por el célebre artista holandés de imágenes imposibles e ilusiones ópticas Bruno Ernst (uno de los principales estudiosos y difusores de la obra de M. C. Escher). Además, se incluyeron una selección de cintas U-matic originales ${ }^{4}$ de las películas de Toti.

La segunda cápsula, Avanzada Digital, es un recuento de los festivales, organizados desde 1998 hasta 2003, y que generaron una plataforma de producción y difusión del video y arte electrónico, y que incorporó también a la música electrónica. Para esto, se expusieron los afiches del Festival Internacional de Video/Arte/Electrónica, desde la segunda edición (1998) hasta la edición sétima $(2003)^{5}$. En una vitrina se presentaban invitaciones

2 ATA. (1995). Gianni Toti: retrospectiva electrónica. Recuperado de: http://bit.ly/GiToti

3 ATA. (1998). Arte y ciencia: centenario de M. C. Escher. Recuperado de: http://bit.ly/MCEscher100

4 Formato de cintas de video utilizadas profesionalmente en los años noventa.

5 A partir del 8. ${ }^{\circ}$ Festival Internacional de Video/Arte/Electrónica, este fue gestionado por Realidad Visual. 
hasta documentos y cartas importantes vinculadas con la organización de las seis ediciones organizadas por ATA y su vínculo histórico con el VIII Festival Internacional de Video Arte organizado por Jorge Glusberg y Alfonso Castrillón. Adicionalmente, se contó con un espacio dedicado a presentar documentación visual y sonora de MODULAR $12^{\circ} 06^{\prime} \mathrm{S}$ $77^{\circ} 01^{\prime}$ W Park-O-Bahn ${ }^{6}$, uno de los primeros eventos de arte sonoro en el Perú, que fue organizado como parte del 5. ${ }^{\circ}$ Festival (2001). Finalmente, se presentó un video con diversos materiales, principalmente extraídos de programas de televisión y registros realizados a lo largo de las seis ediciones del Festival Internacional de Video/ Arte/Electrónica que fueron organizadas por ATA.

El intercambio global de manera justa y crítica, dando visibilidad a artistas peruanos y latinoamericanos, pero también compartiendo experiencias a nivel local, invitando a creadores e intelectuales de diferentes partes del mundo, ha sido un eje fundamental en la trayectoria de ATA. Órbita contiene una selección de tales intercambios. Para esto, se dio espacio a registros en video, como el

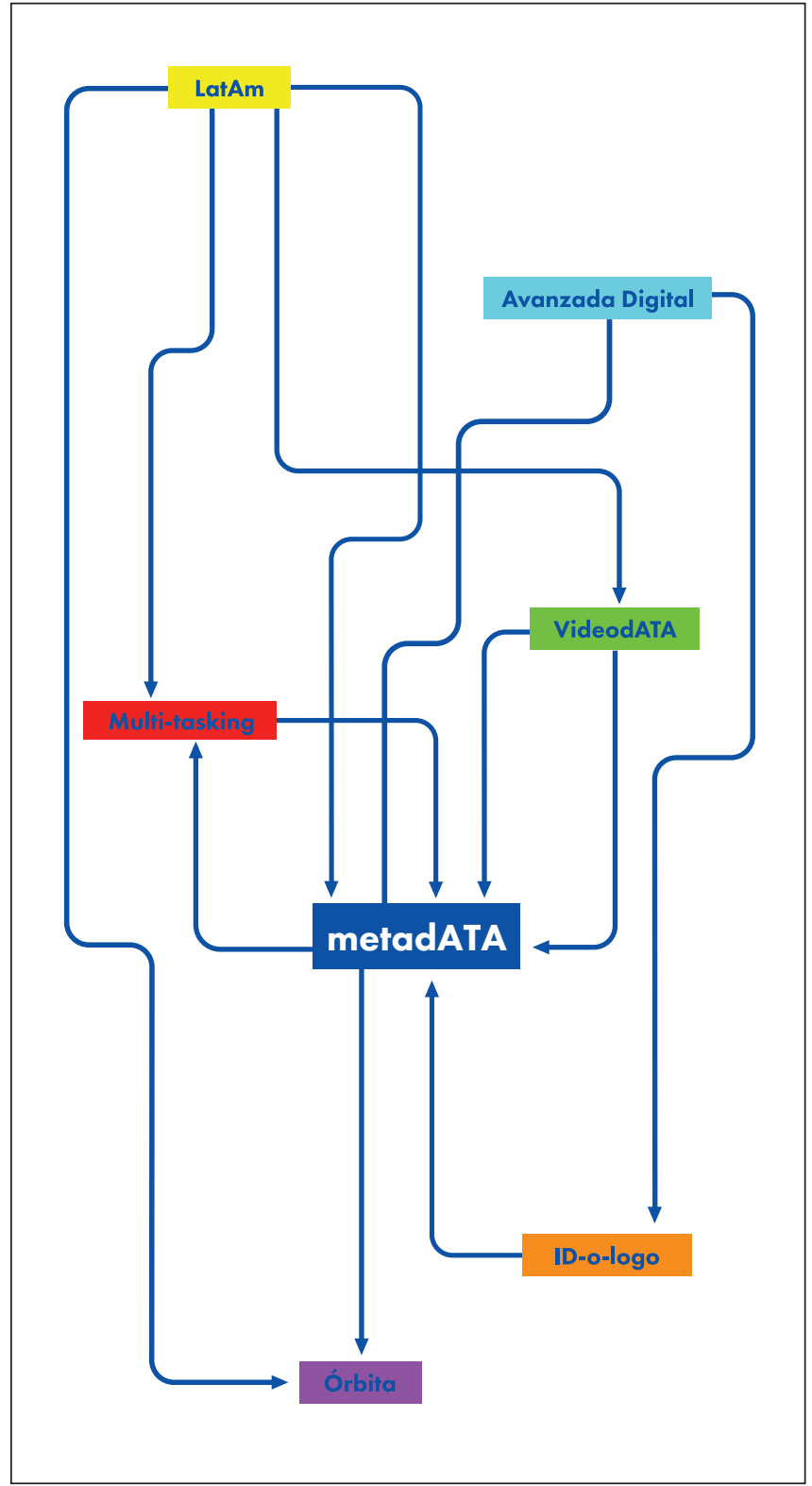

Fig. 4. MetadATA [muestra antológica] . Sala Raúl Porras Barrenechea. Visita junio del 2016. Foto: IS Creative Studio.

primer proyecto llevado a cabo en el Perú por el artista peruano radicado en Bélgica Jota Castro, La palabra de los mudos (2008) ${ }^{7}$, y la instalación interactiva Lima: All the Republic in One $(2012)^{8}$, de José Carlos Martinat, presentada en la Novena Bienal de Shanghái. Otro monitor

6 Modular $12^{\circ} 06^{\prime} \mathrm{S} 77^{\circ} 01^{\prime} \mathrm{W}$ Park-o-Bahn en http://bit.ly/Park-o-Bahn168.

7 ATA. (2008). La palabra de los mudos. Un proyecto de Jota Castro. Recuperado de: http://bit.ly/palabramudos

8 ATA. (2012). Novena Bienal de Shanghái - Lima: All the Republic in One. Recuperado de: http://bit.ly/JCMartinat 


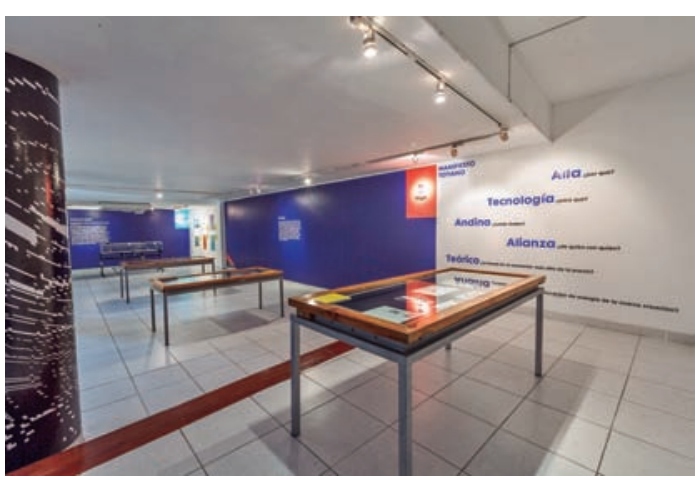

Fig. 5. MetadATA [muestra antológica]. Sala Raúl Porras Barrenechea. Visita junio del 2016. Foto: Wilber Dueñas. incluía el trabajo Cuando la fe mueve montañas, de Francis Alÿs, para la III Bienal de Lima (2002), y The Colony (2016), de Dinh Q. Lê. Una última pantalla presentaba la instalación Zero to Infinity (2013), de Rasheed Arareen, en su versión adecuada para Lima9 .

En el panorama local se trabajó en modo multitasking, es decir, desde múltiples frentes y en simultáneo, desarrollando significativas colaboraciones con organizaciones y personas. En este caso, se brindó un espacio principal a dos proyecciones: la presentación de Stelarc en la Sala de Actos de

la Municipalidad de Miraflores (2001) y la primera performance online en el Perú, titulada No Logo (2001), de Angie Bonino, como parte de la 30 . Edición del Festival Internacional de Nuevo Cinema y Nuevos Medios de Montreal10. Otro video incluía un compilatorio del evento Música electrónica presentado por Johannes Goebel $(2000)^{11}$ en el Goethe Institut Lima, una edición breve de las obras peruanas presentes en el Festival Interferences en Belfort, bajo el título de Perú PER Muta $(2002)^{12}$, y un reportaje a Antoni Muntadas sobre la muestra Espacios protegidos, espacios públicos, organizada en el Centro Cultural de España $(2000)^{13}$.

Al mismo tiempo, se trabajó una vitrina especial con documentos de una serie de actividades de producción y difusión con diversos catálogos y documentos, como: Videografías in(visibles), una selección de video arte latinoamericano 2000-200514; VideoZone2: Bienal Internacional de Video Arte de Tel Aviv (2004) ${ }^{15}$ (que incluyó una selección de video latinoamericano); VíaSatélite: Panorama de la fotografía y el video del Perú contemporáneo ${ }^{16}$, muestra que itineró en América Latina; Mash Up Arte en choque fuga (2010) ${ }^{17}$; y, PerúRemix, antología de video arte peruano en Gdansk (Polonia) ${ }^{18}$. Además, se resaltaba en monitores una selección de las exhibiciones organizadas año 2006, fruto de la colaboración con el Espacio Fundación Telefónica en Lima: VIBRA, Audio Lima Experimental, Festival de Arte Sonoro $(2006)^{19} ;$ BLIP! Robótica del Reciclaje $(2007)^{20}$; VideoJuegos, historias lúdicas, aventuras insólitas $(2009)^{21}$; VIDEO XXI: Colección Lemaitre (2010) ${ }^{22}$; McLuhan 100, comunicaciones, arte y pen-

9 ATA. (2013). Rasheed Araeen en Lima. Recuperado de: http://bit.ly/RasheedA

10 ATA. (2001). Angie Bonino: No logo. Recuperado de: http://bit.ly/ABonino

11 ATA. (2000). Música electrónica: Johannes Goebel en Lima. Recuperado de: http://bit.ly/JGoebel

12 ATA. (2000). Interferences International Electronic Art Festival at Belfort. Recuperado de: http://bit.ly/ InterferencesIE

13 ATA. (2000). Espacios protegidos / Espacios públicos de Antoni Muntadas. Recuperado de: http://bit.ly/esppropu

14 ATA. (2005). Videografías Invisibles. Recuperado de: ver: http://bit.ly/videografiasinv

15 ATA. (2004). VideoZone2. Recuperado de: ver: http://bit.ly/videozone2

16 ATA. (2005). Vía Satélite. Recuperado de: ver: http://bit.ly/viasatelitea

17 ATA. (2010). MASH UP. Arte en choque. Fuga. Recuperado de: http://bit.ly/mashuparte

18 ATA. (2006). Perú Remix. Sztuka wideo z Peru. Recuperado de: http://bit.ly/peruremix

19 ATA. (2006). Festival de arte sonoro: Vibra / Audio Lima Experimental. Recuperado de: http://bit.ly/vibraart

20 ATA. (2007). BLIP! Robótica de reciclaje. Recuperado de: http://bit.ly/bliprobot

21 ATA. (2009). Videojuegos// Historias lúdicas, aventuras insólitas. Recuperado de: http://bit.ly/videojuegoshist

22 ATA. (2010). Video XXI: Colección Lemaitre. Recuperado de: http://bit.ly/videoxxi 
samiento en el centenario de Marshall McLuhan (2011)23; y Arte y óptica: la velocidad de las comunicaciones $(2013)^{24}$.

La cápsula LatAm presenta un recuento de Escuelab, un espacio en el Centro de Lima que, por casi un quinquenio, buscó incentivar a creadores de la región andina y centroamericana a proyectar ideas transdisciplinarias utilizando nuevas tecnologías en los campos del arte, la cultura, la educación y la sociedad. Fue un reto no menor poder ejemplificar todo el trabajo generado en Escuelab en cinco años de funcionamiento; por lo tanto, se diseñaron una serie de gigantografías con los diferentes proyectos de los residentes de Escuelab, así como una selección de las principales actividades. También se pusieron a disposición del público dos computadoras con videos de proyectos asociados con Escuelab y con el proyecto de Insulares/Divergentes: Hacia una nueva cultura tecnológica en América Latina, que prospectó más de un centenar de proyectos de arte, cultura, tecnología y sociedad en la región y que fue un hito fundamental de estudio y análisis ${ }^{25}$.

Finalmente, en la sala 770 presentamos videodATA, un gran repositorio de videos con cerca de doscientos trabajos en los que ATA colaboró en la producción y difusión de manera activa, así como Videografías in(visibles), la primera selección de videoarte que cubrió por completo el horizonte de América Latina. Para hacer este material accesible en computadoras, se implementó un servidor de video en demanda que proveía del acceso a dichos materiales, así como dos pantallas que presentaban una selección de ambos programas con el objetivo

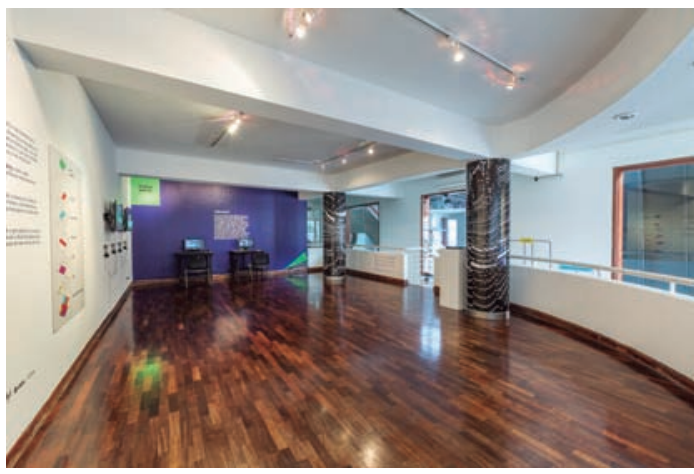

Fig. 6. MetadATA [muestra antológica]. Sala 770. Visita junio del 2016. Foto: Wilber Dueñas.

de despertar curiosidad al público visitante, para que luego pudieran interactuar con los videos disponibles en el repositorio (Fig. 6)

\section{Museografía y diseño}

Para explicar la importancia de la museografía y el diseño en la realización de MetadATA, es relevante primero señalar las condiciones de las salas.

La primera está ubicada en el primer piso del Centro Cultural Ricardo Palma de la Municipalidad de Miraflores. Esta sala, llamada Sala 770 por situarse en la avenida Larco 770 , recibe proyectos de una variedad de artistas jóvenes contemporáneos que buscan tener un espacio de experimentación íntimo y personal. Por un lado, las dimensiones eran relativamente pequeñas $(9,70 \mathrm{~m} \times 4,74 \mathrm{~m})$ para la magnitud de información que poseía el proyecto, lo que nos llamaba a darle un rol claro y diferencial en la muestra. Además de esto, poseía un condicionante en la iluminación natural a causa de un gran ventanal que daba a la vía pública y ocupaba las dimensiones de toda una pared, lo que podía convertirse en un problema al ser una muestra que consistía principalmente de material en video. Asimismo, el espacio poseía un balcón que miraba hacia el interior del edificio. Desde allí se podía ver la sala de exposición del sótano, a manera de ayuda para buscar una forma de integrar ambos espacios como uno solo.

23 ATA. (2011). MMcLuhan 100: comunicaciones, arte y pensamiento. Recuperado de: http://bit.ly/MMcLuhan100

24 ATA. (201). Arte y óptica: la velocidad de las comunicaciones. Recuperado de: http://bit.ly/arteyoptica

25 Insulares Divergentes. (2009). Insulares Divergentes. Recuperado de: http://bit.ly/insularesdiv 


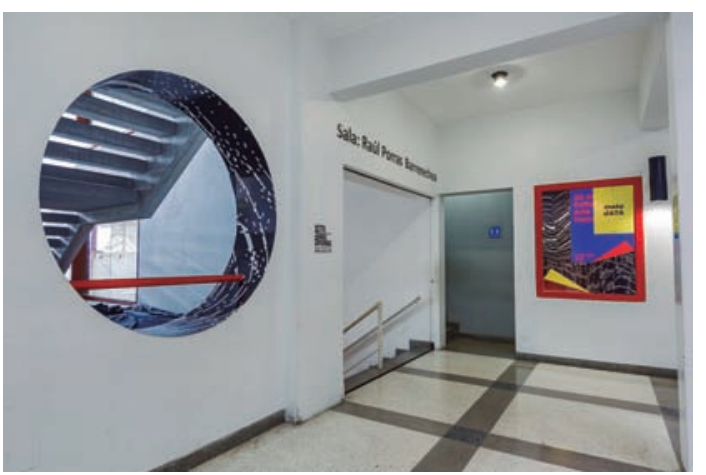

Fig. 7. MetadATA [muestra antológica] . Sala Raúl Porras Barrenechea. Visita junio del 2016. Foto: Wilber Dueñas.
La segunda sala, llamada Raúl Porras Barrenechea en honor al célebre historiador y político liberal afincado en Miraflores (Fig. 7), como ya comentamos, se ubica en el sótano del mismo Centro y recibe a artistas más reconocidos y muestras colectivas. Un punto a favor fue que este espacio contaba con dimensiones amplias $(17 \times 15 \mathrm{~m}$ aprox.), que nos daban la flexibilidad de organizar en esta área buena parte del contenido seleccionado para la muestra. Sin embargo, su ubicación en el subsuelo del centro cultural podía hacerla un tanto inaccesible.

Debíamos generar un recorrido entre ambas salas para que los visitantes transitaran por toda la muestra. Esto implicaba una tarea adicional, ¿cómo lograr que todo el recorrido no sea extenso ni tedioso para el visitante? Debíamos decidir de qué forma dividir la información y cómo darle personalidad dentro de la propuesta curatorial. Adicionalmente a esto, la estructura interna de ambos espacios era el reto no menor, ya que teníamos que considerar en el diseño una serie de columnas, así como puertas de oficinas y depósitos existentes dentro de los ambientes expositivos. Estos son residuos de espacios que originalmente no fueron diseñados como salas de exhibición y que, por lo tanto, nunca tuvieron un plan integral de remodelación que permitiera lograr una arquitectura flexible acorde a las exposiciones que buscan albergar.

En consecuencia, ambos espacios tienen problemáticas diferentes que la muestra debía sortear. Quizá fueron dos retos los mayores dentro del trabajo en el espacio de exposición: primero, mantener una consistencia visual a lo largo de toda la muestra, pese a las estructuras heterogéneas y residuales existentes como parte de la arquitectura misma de las salas; y, segundo, unificar ambas salas para que dieran la apariencia de ser un solo gran espacio en donde se permitiera generar un flujo coherente para los visitantes, y que a su vez permitiese discurrir por las seis cápsulas de información planteadas de manera simple y didáctica, sin que fuera necesario obligar a los visitantes a seguir un recorrido lineal o consecutivo.

Para el diseño museográfico y el montaje, se armaron varios planos con diferentes posibilidades que nos permitieran aprovechar el espacio de las dos salas. El objetivo era integrar ambas zonas, crear islas para cada cápsula de información y dar algunas herramientas transversales que permitieran facilitar y asimilar el recorrido. Es así que las zonas de visualización de obras (videodATA) en el primer piso (sala 770) y la cronología ubicada en el subsuelo (sala Raúl Porras Barrenechea) actuaban como elementos transversales o anclas ${ }^{26}$, que permitían dar miradas transversales a muchos de los hitos de información presentes en el recorrido.

Para explicar cómo se aprovecharon y resolvieron cada uno de los espacios, debemos antes detallar los requerimientos para la construcción de la identidad de la muestra, que se resumían en la necesidad de transmitir la estética del video arte en una identidad sólida, pero

26 El término ancla es muy frecuentemente utilizado para describir en los shopping malls las grandes tiendas por departamento existentes a cierta distancia una de la otra y entre las cuales el visitante transita por un gran número de tiendas pequeñas. Este mismo concepto se aplica muchas veces en muestras en donde algunas grandes piezas o estaciones con temas centrales se ubican claramente diferenciadas de los que podría ser el resto de las obras. 
que brindara espacio a cada una de las cápsulas de información que contenía la muestra. El gran reto siempre fue siempre darle coherencia a la gran cantidad de información y la necesidad de estructurar gráficamente el itinerario del público para facilitar el entendimiento del contenido, considerando lo variado de la audiencia y, por lo tanto, las diferentes necesidades en términos de búsqueda e indagación de información, es decir, tomando en cuenta que la muestra debía ser útil tanto para profesionales del arte (artistas, curadores, investigadores), así como para público interesado en temas de ciencia y tecnología y, finalmente, público en general que pudiese percibir el contenido como cercano.

La propuesta visual de identidad propuesta por IS Creative Studio jugaba con una tríada de colores representativos del video (azul, amarillo y rojo) y la textura característica del ruido en la transmisión del video analógico, pero sumado a elementos estéticos propios del lenguaje digital. También se utilizaron gráficamente las figuras de documentos superpuestos para reforzar la idea de repositorio de información al que hacía referencia metadATA. Asimismo, se utilizó de apoyo una paleta de colores que le diera carácter propio y permitiera la fácil identificación de cada una de las cápsulas de información que conformaban la exposición: ID-o-logo en naranja, Avanzada Digital en celeste, Órbita en rosado, Multitasking en rojo, LatAm en amarillo y VideodATA en verde. Estos códigos gráficos se emplearon para el espacio y el material impreso.

Como parte de la propuesta visual de la muestra, se contaron con algunos diseños que ayudaron a estructurar la información recopilada en la investigación y que servían como herramientas transversales a toda la exposición facilitando el recorrido y la unión visual de toda la información. Como ejemplo de

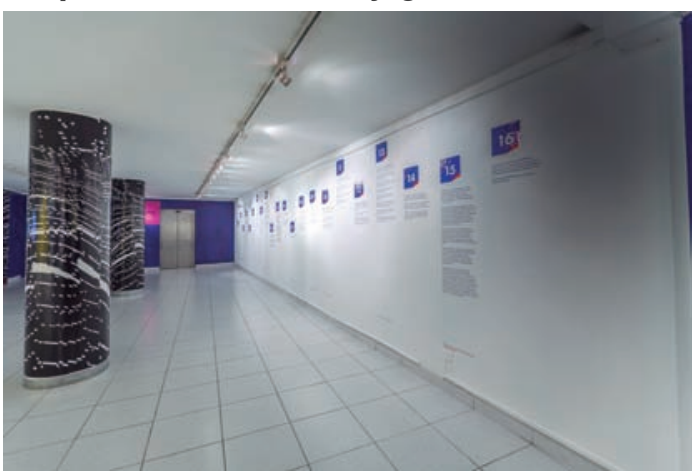

Fig. 8. MetadATA [muestra antológica] . Sala Raúl Porras Barrenechea. Visita junio del 2016. Foto: IS Creative Studio.

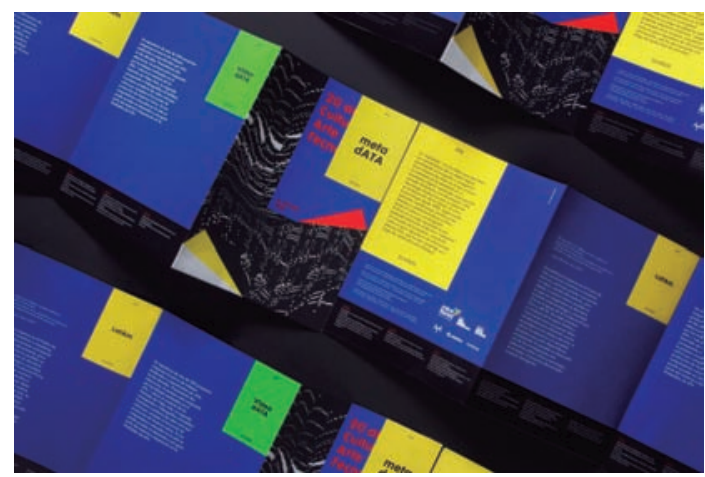

Fig. 9. MetadATA [folleto]. Foto: Wilber Dueñas. esto, tenemos el mapa conceptual de todas las cápsulas que integraba las dos salas; la cronología desde 1995 hasta 2016, que exponía los hitos de ATA (Fig. 8) con algunos datos adicionales sobre la historia de la tecnología, y el diagrama de las salas que buscaba mostrar espacialmente cómo se habían distribuido los temas dentro del espacio.

La propuesta realizada por IS Creative Studio (Fig. 9) ha sido presentada como referente en el diseño de muestras en reconocidos eventos internacionales como Welformat Poster Festival (Suiza, 2016), Typomania Festival (Rusia, 2017), Graphic Design Festival (Escocia, 2017) y el Graphic Design Festival (Seoul, 2017), además de haber sido seleccionado por el prestigioso portal Behance para su sección de Diseño de Exposiciones ${ }^{27}$.

27 metadATA - 20 años de Cultura, Arte y Tecnología (ver: http://bit.ly/behancemet) 


\section{Dispositivos tecnológicos y nuevos contenidos en video}

Al tener una considerable parte del material del archivo de ATA digitalizado, la oportunidad era propicia para recombinar y catalogar el material existente de nuevas formas que permitieran la creación y producción de conocimiento relevante y actual para la muestra. Por ello, la exhibición incluyó una cantidad importante de materiales audiovisuales, tanto compilaciones editadas a partir del material en archivo, como videos disponibles en terminales para la consulta a partir de un buscador en la base de datos de ATA.

El trabajo de producción de nueve contenidos en video requirió de la revisión exhaustiva de horas del material, para así hacer un pietaje y definir qué partes podrían ser relevantes de presentar, tomando en consideración los temas de cada cápsula de información. Es decir, no se trató de una edición de material antiguo uno tras de otro, sino que se concibieron guiones basado en el pietaje seleccionado para estructurar el material audiovisual en nuevos videos que permitan ilustrar y contextualizar históricamente la muestra.

Por otro lado, ATA cuenta con diversos títulos de la producción nacional e internacional, aparecidos en los últimos treinta años, que forman parte de su colección. Una parte importante de esta colección corresponden a obras de video arte peruano, principalmente producidos entre fines de los noventa e inicios de la primera década del siglo XXI, que habitualmente solo son disponibles para consulta, con la previa autorización de los autores. Fig. 10. Como ya lo hemos mencionado, en esta oportunidad, la mayoría de estas obras peruanas estuvieron disponibles en terminales debidamente indexadas, lo que permitía su búsqueda en la base de datos que las relacionaba entre sí y que permitía a los públicos que no conocían estos trabajos poder navegar en un universo de información audiovisual similar a las formas en que hoy buscamos información o accedemos a videos en Internet.

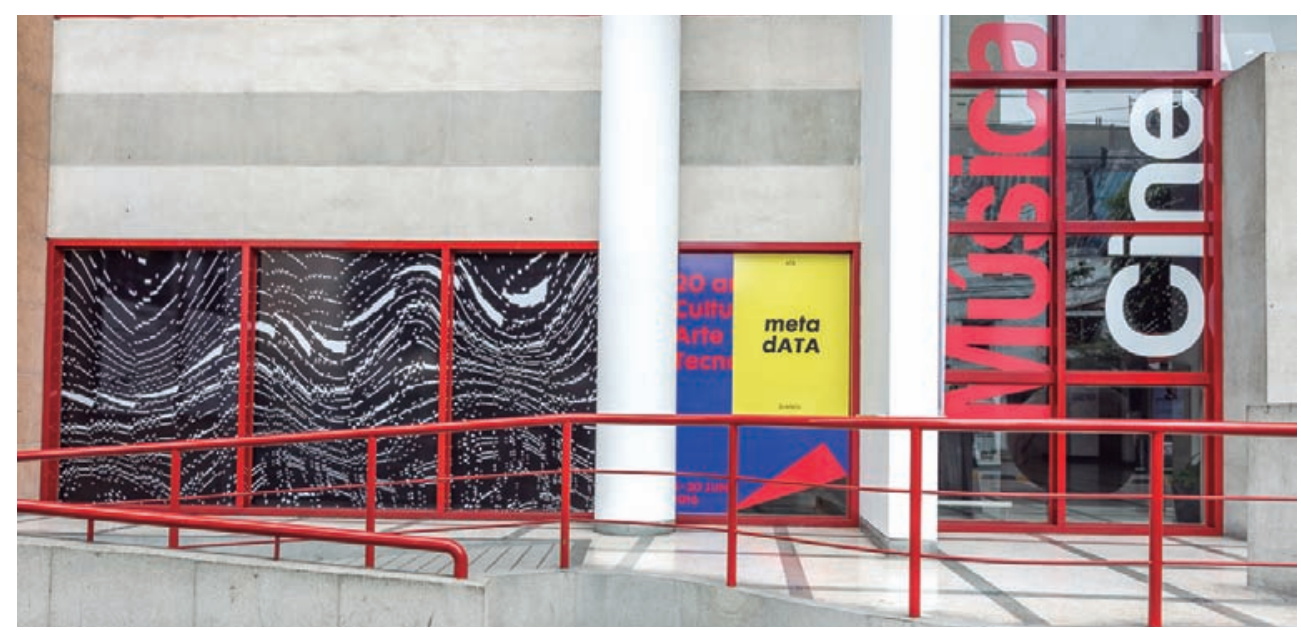

Fig. 10. MetadATA [muestra antológica]. Sala 770. Visita junio del 2016. Foto Wilber Dueñas.

\section{Epílogo: (re)curaduría, memoria y archivo}

Como ya lo hemos mencionado, MetadATA fue una muestra que buscaba hacer una exégesis del trabajo que hicimos durante veinte años; por lo tanto, tiene las características de una muestra retrospectiva o revisión histórica, pero mediante un análisis objetivo. Sin embargo, existen problemáticas en relación con la objetividad de este tipo de curadurías, considerando que involucran trabajo previamente curado por algunas de las mismas personas que también participaron en la curaduría de MetadATA. 
Es decir, una problemática en este tipo de curadurías radica en la poca autocrítica al trabajo conducido previamente y hasta qué punto consideramos que dicho trabajo fue relevante en el pasado de cara a la contextualización en el presente. Uno se topa con perspectivas históricas disímiles a las que originalmente propiciaron las actividades o proyectos realizados. Si bien los trabajos deben enmarcarse en un tiempo determinado, uno de los mayores retos consiste en recoger la emoción y las razones por las que se realizó un proyecto en un determinado momento y analizarlo desde una perspectiva actual. Toda vez que la tecnología va evolucionando y muchas de las técnicas de los últimos veinte años han caducado, debemos extraer el contenido relevante detrás de la necesidad en el uso de dichos dispositivos técnicos, lo que no es un proceso fácil, pues se deben articular elementos como la memoria, la curaduría y el espacio en donde se desarrolló el proyecto (sea en una sala de exhibición, un parque, una sala de proyección, u otro espacio, y tanto en el Perú como el extranjero). Considerando que, fuera del trabajo de los documentos originales del archivo, los demás materiales de la muestra corresponden a objetos desmaterializados en forma de videos y bases de datos, estos nos podrían enfrentar a la pregunta de por qué hacer una muestra de este tipo en un espacio físico y si hasta resulta pertinente para mostrar este tipo de obras. Lo interesante del trabajo que se hizo en las Salas del Centro Cultural Ricardo Palma, pese a los retos que este implicaba, fue que permitió ofrecer al visitante un recorrido u orientación a partir de las cápsulas de información y la cronología. Las cronologías son herramientas que nos ayudan a poner en contexto el tiempo y relacionarlo con otros hitos históricos y situaciones conexas. La cronología nos dio estructura para articular la muestra. Construir la cronología de ATA nos permitió también hacer una selección de los documentos y proyectos más relevantes, de cara a definir una memoria histórica y crítica de las primeras dos décadas de ATA. También fue muy útil que el equipo curatorial incluyese a dos integrantes jóvenes (Nataly Montes y Reina Jara) que no habían participado en muchos de los proyectos presentados y que, por lo tanto, no tenían preconceptos parcializados y fomentaban un diálogo intergeneracional en el equipo.

MetadATA resume un momento cultural y una emoción propia al establecimiento de la creación de video y nuevos medios en el Perú. El conjunto seleccionado del archivo de ATA y articulado en cápsulas nos permitieron contar una historia de esta época que busca capturar dicha emoción e identificarla con momentos y situaciones que permitan integrar y relacionar la historia de las artes visuales en el Perú con muchas otras historias de prácticas multidisciplinares.

\section{Créditos}

metadATA: 20 años de cultura, arte y tecnología

Organizado por ATA, Municipalidad de Miraflores, Sala 770 y Sala Raúl Porras Barrenechea del Centro Cultural Ricardo Palma

Fecha: del 3 al 30 de junio del 2016

Curaduría: José-Carlos Mariátegui, Jorge Villacorta, Nataly Montes B. y Reina Jara

Diseño de sala: Richars Meza, Rommina Dolorier y María Boada (IS Creative Studio)

Guiones de videos: Maia Rojas

Edición de video: Kent Salas

Director Salas 770 y Raúl Porras Barrenechea: Rubén Ramos

Iluminación y montaje: Luis Oqueliz y Jairo Palomino

Agradecimientos: Wilber Dueñas, José Javier Castro, Kiko Mayorga, Ysabel Tunque, Ana Torres, Néstor Escurra y Elvis Carnaqué 